\title{
Criteria for Determining Malignancy in Pancreatic Intraductal Papillary Mucinous Neoplasm Based on Computed Tomography
}

\author{
Peter Mönnings $^{\mathrm{a}}$ Orlin Belyaev ${ }^{\mathrm{b}}$ Waldemar Uhl ${ }^{\mathrm{b}} \quad$ Arnd Giese $^{\mathrm{c}}$ \\ Andrea Tannapfel ${ }^{d}$ Odo Köster ${ }^{a}$ Juris J. Meier ${ }^{c}$ \\ ${ }^{a}$ Department of Diagnostic and Interventional Radiology, ${ }^{\mathrm{b}}$ Department of Surgery, and ${ }^{\mathrm{c}}$ Diabetes Division, \\ Department of Medicine I, St. Josef-Hospital, and ${ }^{d}$ Department of Pathology, Bergmannsheil, Ruhr-University \\ Bochum, Bochum, Germany
}

\section{Key Words}

Intraductal papillary mucinous neoplasm - Computed

tomography $\cdot$ Sensitivity $\cdot$ Specificity $\cdot$ Malignancy

\begin{abstract}
Introduction: Determining the dignity of intraductal papillary mucinous neoplasms (IPMNs) by imaging procedures is challenging. Various CT-based criteria were evaluated. Patients and Methods: Preoperative CT scans from 47 patients with IPMN were analyzed. Predefined criteria of malignancy were compared between patients with benign (bIPMN; $n=28$ ) and malignant (mIPMN; $n=19$ ) tumors, and a summation score was determined. Results: Preoperative carbohydrate-antigen 19-9 levels were higher in patients with $\operatorname{mIPMN}(p=0.013)$. The diameter of the main pancreatic duct was greater in patients with mIPMN $(p<0.0001)$. More patients with mIPMN showed bile duct obstruction ( $p=0.0076)$, solid tumor components $(p=0.0076)$, contrast enhancement in cystic walls ( $p=0.0086)$, peripancreatic lymph nodes ( $p=0.0076)$, and abrupt diameter changes of the main pancreatic duct $(p=0.0008)$. The CT density of the
\end{abstract}

\section{KARGER}

(c) 2016 S. Karger AG, Basel

E-Mail karger@karger.com

www.karger.com/dig cysts was higher in mIPMN ( $p=0.0063)$. The diagnostic accuracy of the summation score (sensitivity: 0.84 , specificity: 0.96 ) was greater when compared to each individual CT parameter. Conclusions: The prevalence and extent of various CT-based abnormalities are greater in patients with mIPMN, but the wide overlap limits the diagnostic value of each individual parameter. A simple summation score largely enhances the diagnostic accuracy.

(c) 2016 S. Karger AG, Basel

\section{Introduction}

Intraductal papillary mucinous neoplasms (IPMNs) are cystic formations of the pancreas originating from the ductal tree [1]. In recent years, the prevalence of diagnosed IPMN has increased substantially [2], owing to the growing availability of imaging techniques with high local resolution of pancreatic tissue, such as X-ray CT, MRI, and endoscopic ultrasound (EUS). Indeed, intrapancreatic cystic lesions are incidentally detected in $\sim 1-3 \%$ of all CT or MRI examinations $[3,4]$. This increasing num- 
ber of incidental diagnoses of cystic pancreatic tumors poses the problem of determining the dignity of the lesions. Thus, whereas a majority of serous cystadenomas of the pancreas are benign, malignancy occurs in most mucinous cystadenomas and IPMN over time [1]. Furthermore, an adenoma-to-carcinoma sequence with increasing levels of cell dysplasia has been proposed for the natural history of IPMN, analogous to the carcinogenesis model of colorectal tumors [5].

In light of the high risk of malignant transformation of IPMN, surveillance strategies have been developed to help in making a decision of whether to surgically remove the tumors or whether to monitor them $[6,7]$. These involve the evaluation of the pancreatic lesions using EUS, MRI, and CT scans. Although the diagnostic value of MRI and EUS appears to be superior, abdominal CT scans are still the standard procedure [8], given the broad availability of this technique. Therefore, it is important to define criteria that allow for an accurate distinction between benign and malignant IMPN based on abdominal CT scans.

A number of individual criteria have been used to distinguish malignant from benign IPMN. Most importantly, the involvement and dilation of the main pancreatic duct as well as the diameter of the cystic tumor formations have been used as criteria for ascertaining malignancy $[6,9-12]$.

Indeed, pancreatic surgery is usually recommended in the case of main pancreatic duct involvement or in lesions greater than $30 \mathrm{~mm}$ in diameter with high-risk stigmata based on the latest "International Guidelines" [6]. Additional parameters that have been associated with malignant tumor behavior include the obstruction of the bile duct, presence of solid tumor components, contrast enhancement of cystic walls, distal pancreatic atrophy, enlarged peripancreatic lymph nodes, abrupt changes in the diameter of the main pancreatic duct, and the presence of tumor calcifications.

However, even though all of these parameters have been associated with an increased risk of malignancy, they do not allow for a clear-cut distinction between benign and malignant lesions, when determined on an individual basis.

Therefore, the present study was designed to determine the diagnostic sensitivity and specificity of different CT-based parameters for the detection of malignant IPMN. Furthermore, a summation score consisting of different components was developed in order to enhance the diagnostic accuracy of CT-based parameters for the detection of malignant IPMN.

CT Criteria for Malignant IPMN

\section{Patients and Methods}

\section{Study Protocol}

A retrospective analysis of abdominal CT scans was performed in patients who had undergone surgery for the removal of pancreatic IPMN's. Data were obtained from a retrospective chart review of all patients undergoing abdominal CT scans at the Department of Radiology, St. Josef-Hospital, Ruhr-University Bochum, between 2006 and January 2013. All analyses were performed in a blinded fashion after pseudonymization of patient-level data. The study protocol was approved by the Ethics Committee of the medical faculty of the Ruhr-University Bochum.

\section{Patients}

A total of 96 patients had undergone pancreatic surgery for the removal of pancreatic IPMN at the Department of Surgery, St. Josef-Hospital Bochum, between 2006 and January 2013. Among those, abdominal CT scans were available for analysis from $47 \mathrm{pa}-$ tients. The diagnosis of pancreatic IPMN was confirmed in all cases by histopathological analysis performed by an experienced pathologist using established criteria [13]. CT scans were performed at the Department of Radiology, St. Josef-Hospital Bochum, in 44 patients, and by external Radiologists in 3 cases. The technical quality and comparability of these external CTs with internal CTs were assured prior to inclusion of these images. To determine potential differences in various anthropometric, clinical chemistry, and CT-based parameters, patients were divided in 2 different groups according to the histopathological evaluation: (A) 28 patients (59.6\%) with benign IPMN and (B) 19 patients (40.4\%) with malignant IPMN.

\section{Patient Characteristics and Laboratory Determinations}

The following parameters were recorded from the patient records: age, gender, carbohydrate-antigen 19-9 (CA 19-9), bilirubin, amylase, lipase, alkaline phosphatase gamma glutamyltansferase (GGT), and fecal elastase. The most recent preoperative laboratory values were used for the analyses. In 1 patient, laboratory values for CA 19-9, bilirubin, amylase, lipase, and GGT were not available. In 10 patients, fecal elastase levels were not available. Furthermore, a history of known diabetes and of exocrine pancreatic insufficiency was recorded from the patient charts.

\section{CT Scan Protocol}

All CT-scans were performed on a 16-slice-CT scanner (Somatom 16, Siemens, Erlangen, Germany). The protocol for pancreatic CT included non-enhanced abdominal CT, abdominal arterial phase $\mathrm{CT}$, and portal-venous phase CT. In patients without a known history of pancreatic abnormalities, only standard abdominal CTs without non-enhanced phases were performed $(n=11)$.

All patients were asked to drink an oral iodine-containing contrast agent $(30 \mathrm{~mL}$ with a concentration of $400 \mathrm{mg}$ iodine $/ \mathrm{mL}$ diluted in $1 \mathrm{~L} \mathrm{H}_{2} \mathrm{O}$ ), starting approximately $1 \mathrm{~h}$ before CT-examination. Iomeprol 300 (300 mg iodine $/ \mathrm{mL}$ ) was injected as an intravenous contrast agent at a rate of $3 \mathrm{~mL} / \mathrm{s}$. The total volume of injected contrast agent was $100 \mathrm{~mL}$. One hundred twenty kilovolts voltage was applied during all phases. Collimation was $16 \times 1.5 \mathrm{~mm}$ in non-enhanced CTs and $16 \times 0.75 \mathrm{~mm}$ in arterial and portalvenous phases. The pitch was 0.75 . Multi-planar reformations were done in a coronal and a sagittal plane. Moreover, curved reformations along the main pancreatic duct were performed. 
Table 1. Characteristics and laboratory parameters in patients with benign and malignant IPMN

\begin{tabular}{lccl}
\hline Parameter & Malignant IPMN & Benign IPMN & $p$ value \\
\hline Age, years & $62.2 \pm 2.4$ & $64.8 \pm 1.7$ & 0.36 \\
Gender, female/male & $10 / 9$ & $12 / 16$ & 0.56 \\
CA 19-9, U/mL & $50.9 \pm 11.4$ & $21.6 \pm 5.4$ & 0.013 \\
Bilirubin, mg/dL & $0.68 \pm 0.11$ & $0.53 \pm 0.04$ & 0.13 \\
Amylase, U/L & $67.1 \pm 22.6$ & $57.2 \pm 17.0$ & 0.72 \\
Lipase, U/L & $147.4 \pm 42.5$ & $110.1 \pm 31.9$ & 0.48 \\
AP, U/L & $73.1 \pm 5.9$ & $78.8 \pm 5.8$ & 0.51 \\
$\gamma$-GT, U/L & $75.7 \pm 27.1$ & $40.0 \pm 7.7$ & 0.14 \\
Faecal elastase, $\mu$ g/g & $291.3 \pm 54.3$ & $287.0 \pm 43.5$ & 0.95 \\
Diabetes (yes/no) & $8 / 10$ & $11 / 16$ & 1.0 \\
Exocrine insufficiency (yes/no) & $4 / 3$ & $27 / 0$ & 0.051 \\
\hline
\end{tabular}

Means \pm SEM; statistics: Student's $t$ test or Fisher's exact test.

In CTs obtained before $2009(n=10)$, only 5 and $10 \mathrm{~mm}$ slices were archived, which did not allow for reformations of the main pancreatic duct.

\section{Criteria for CT Analysis}

All CT scans were retrospectively analyzed for potential criteria of malignancy in IPMN. The analyses were performed on a radiological workstation (JiveX Diagnostic Client, Visus, Bochum, Germany) by an experienced radiologist.

The following parameters were derived from the CT images:

1. Maximum diameter of the main pancreatic duct;

2. Maximum diameter of cystic formations;

3. Maximum diameter of the supra-pancreatic bile duct (Ductus choledochus): a diameter of more than $10 \mathrm{~mm}$ in the absence of other obvious causes of obstruction was considered as evidence of bile duct obstruction;

4. Presence of solid tumor components or mural nodules;

5. Contrast enhancement of cystic walls;

6. Distal pancreatic atrophy: the axial diameters of the pancreatic parenchyma in the tail and in the corpus were determined: a ratio of less than 0.5 was considered evidence for distal pancreatic atrophy. This parameter could not be determined in 1 patient with benign and 1 patient with malignant IPMN due to previous distal pancreatectomies;

7. Presence of peripancreatic lymph nodes with a maximal diameter of more than $10 \mathrm{~mm}$;

8. Abrupt changes in the diameter of the main pancreatic duct of more than $50 \%$;

9. Presence of tumor calcifications;

10. CT density (Hounsfield Units, HU) in the cystic part of the lesion in non-enhanced phases. This analysis could not be performed in 5 patients with malignant and 6 patients with benign IPMN due to lack of non-enhanced images.

Calculations and Statistical Analyses

Results are reported as mean \pm SEM. All parameters were first compared between the patients with benign and malignant IMPN.

Numeric parameters were compared using Student's $t$ test. Parametric parameters were compared using Fisher's exact test. A
2 -sided $p$ value $<0.05$ was considered to indicate significant differences.

Furthermore, summation scores of all CT-based parameters that were significantly different between the groups were calculated, and the respective values for diagnostic sensitivity, specificity, positive predictive value (PPV), and negative predictive value (NPV) of each of these scores for the diagnosis of a malignant IPMN were calculated. All statistical analyses were performed using GraphPad Prism 6.01 (GraphPad Software Inc., 2012, La Jolla, CA, USA).

Finally, a multivariate regression analysis including all CTbased parameters that were significantly different between the groups was performed using Statistica version 5.0 (Statsoft Europe, Hamburg, Germany).

\section{Results}

\section{Patient Characteristics and Laboratory Values}

The group of malignant IPMN comprised 9 male (47.4\%) and 10 female patients (52.6\%). In the group of benign IPMN, there were 16 male (57.1\%) and 12 female $(42.9 \%)$ patients $(p=0.56)$. There was no difference in age between patients with malignant and benign IPMN (62.2 \pm 10.5 vs. $64.8 \pm 8.8$ years; $p=0.36$; Table 1).

Preoperative levels of CA 19-9 were significantly higher in patients with malignant IPMN $(50.9 \pm 11.4$ vs. $21.6 \pm$ $5.4 \mathrm{U} / \mathrm{mL} ; p=0.013$; Table 1$)$. However, there was a large degree of overlap between the groups. None of the other laboratory parameters showed significant differences between the groups of patients with benign and malignant IMPN (Table 1).

The presence or absence of known diabetes could be ascertained in 18 patients with malignant and 27 patients 


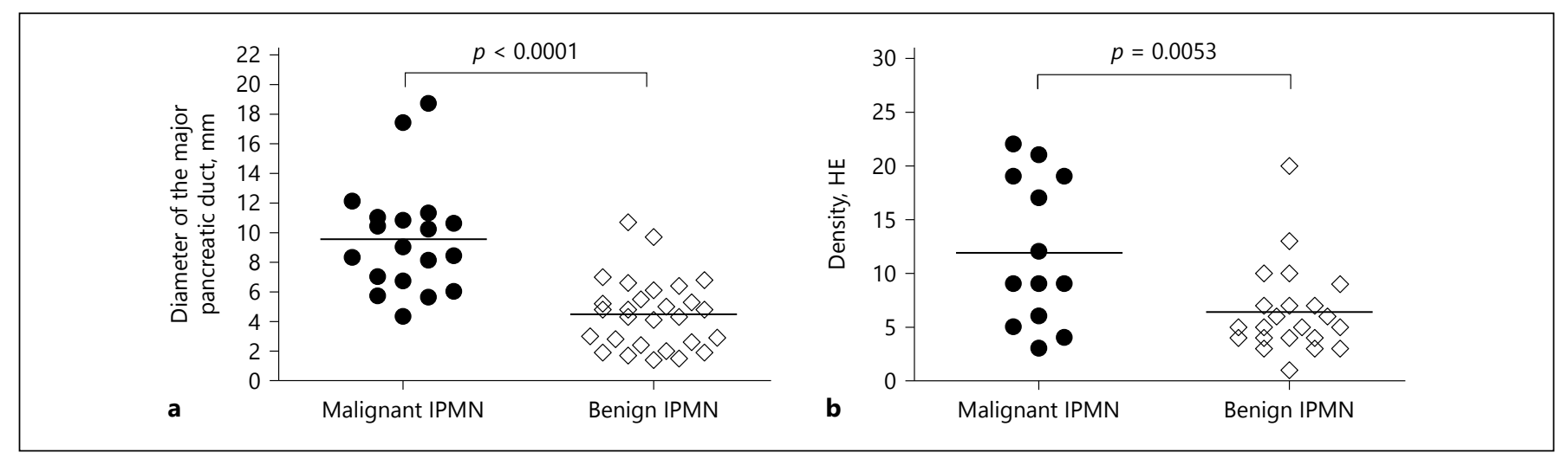

Fig. 1. The mean diameter of the major pancreatic duct (a; determined in 47 patients) and the mean CT density of the cystic components of IPMN in non-enhanced CT (b; determined in 35 patients) in patients with malignant and benign pancreatic IPMN. Data are presented as individual numbers; horizontal lines denote the respective mean values. Statistics were carried out using Student's $t$ test. with benign IMPN. There were no differences regarding the prevalence of diabetes between the groups (42.1 vs. $44.4 \% ; p=1.0$; Table 1).

Information about exocrine pancreatic insufficiency could be obtained from 17 patients with malignant and 27 patients with benign IPMN. The respective prevalence was $82.4 \%$ in patients with malignant and $100 \%$ in patients with benign IPMN $(p=0.051)$.

\section{Comparison of CT-Based Parameters}

Significant differences between patients with benign and malignant IPMN were found for 7 out of the 10 parameters examined.

The mean diameter of the main pancreatic duct was $9.6 \pm 0.9 \mathrm{~mm}$ in patients with malignant IPMN and $4.5 \pm$ $0.4 \mathrm{~mm}$ in patients with benign IPMN $(p<0.0001$; Fig. 1$)$. Ten out of 19 patients (52.6\%) with malignant IPMN exhibited diameters of the main pancreatic duct $>10 \mathrm{~mm}$, and further 8 out of 19 (42.1\%) patients had diameters between 5 and $10 \mathrm{~mm}$ (Fig. 2). In contrast, in patients with benign IPMN, the main pancreatic duct diameter was $>10 \mathrm{~mm}$ in 1 patient (3.6\%), and between 5 and $10 \mathrm{~mm}$ in another 9 patients $(32.1 \%)$. Thus, the parameter "main pancreatic duct diameter $>10 \mathrm{~mm}$ " exhibited a diagnostic sensitivity of 0.5 , a specificity of 0.96 , a PPV of 0.90 , and an NPV of 0.75 for the diagnosis of a malignant IPMN.

A maximum diameter of the supra-pancreatic bile duct of more than $10 \mathrm{~mm}$ in the absence of other obvious causes of bile duct obstruction was found in 5 out of 19 (26\%) patients with malignant and zero out of $28(0 \%)$ patients with benign IPMN ( $p=0.0076$; Fig. 2$)$. Therefore, the diagnostic sensitivity of this parameter for the diagnosis of a malignant IPMN was 0.16 , specificity was 1.0 , the PPV was 1.0 , and the NPV was 0.64 .

Solid tissue components were detectable in 5 out of 19 patients (26\%) with malignant and zero out of $29(0 \%)$ patients with benign IPMN ( $p=0.0076$; Fig. 2 ). Therefore, the sensitivity for the diagnosis of a malignant IPMN was 0.16 , specificity was 1.0 , the PPV was 1.0 , and the NPV was 0.64 .

Contrast enhancement in cystic walls was observed in 8 out of 19 patients (42\%) with malignant and 2 out of 29 (6.9\%) patients with benign IPMN ( $p=0.0086$; Fig. 2). Thus, this parameter exhibited a diagnostic sensitivity of 0.42 , a specificity of 0.93 , a PPV of 0.8 , and an NPV of 0.7 for the diagnosis of a malignant IPMN.

Peripancreatic lymph nodes with an maximum diameter of more than $10 \mathrm{~mm}$ were observed in 5 patients with malignant and $(17.2 \%)$ and no patient with benign (0\%) IPMN ( $p=0.0076$; Fig. 2 ). Therefore, the sensitivity for the diagnosis of a malignant IPMN was 0.26 , specificity was 1.0, the PPV was 1.0 , and the NPV was 0.67 .

Abrupt changes in the diameter of the main pancreatic duct of more than $50 \%$ were found in 7 patients with malignant (36.8\%) and no patient with benign IPMN $(p=$ 0.0008 ; Fig. 2). On that basis, a sensitivity of 0.37 , a specificity of 1.0, a PPV of 1.0, and an NPV of 0.7 for the diagnosis of a malignant IPMN were calculated.

The CT density of the cystic part of the lesions on the non-enhanced images was higher in malignant than in benign IPMN (14.8 \pm 3.4 vs. $6.4 \pm 0.9 \mathrm{HE} ; p=0.0063$; Fig. 1). When a threshold of $10 \mathrm{HE}$ was defined as diagnostic threshold, a sensitivity of 0.5 , a specificity of 0.82 , a PPV of 0.64 , and an NPV of 0.72 were calculated for the detection of a malignant IMPN. 


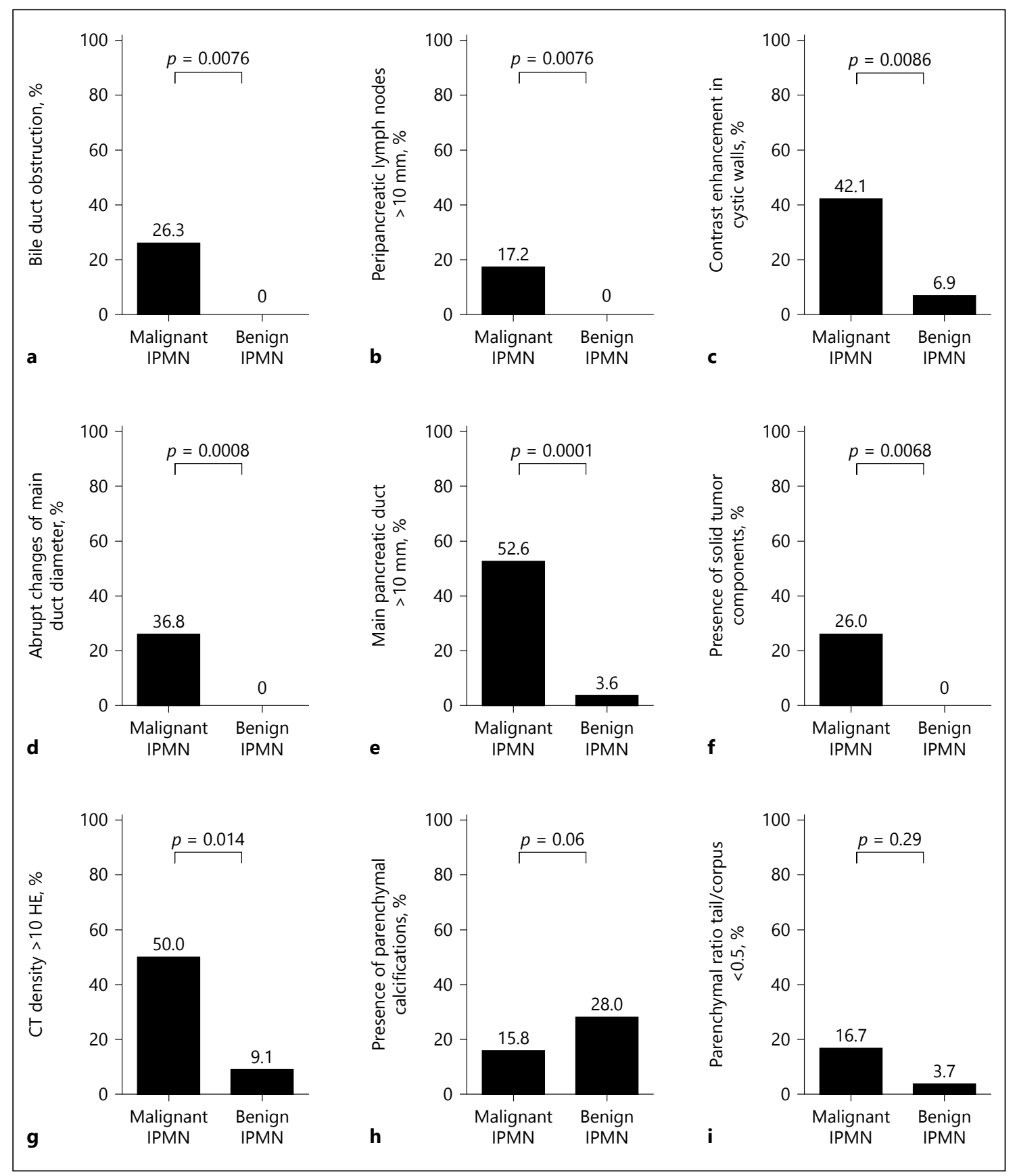

Fig. 2. a-i Different parameters derived from pancreatic CT scans in patients with malignant and benign pancreatic IPMN. Data are presented as percentage numbers of the respective groups. Statistics were carried out using Student's $t$ test.

In contrast to the above-mentioned parameters, the maximum diameter of cystic formations within the lesions was not different between patients with benign and malignant IPMN $(29.4 \pm 3.9$ vs. $36.7 \pm 5.2 \mathrm{~mm}, p=0.25$; details not shown). There were also no differences in the presence of tumor calcifications between patients with malignant $(3 / 19 ; 15.8 \%)$ and benign $(0 / 28 ; 0 \%)$ IPMN $(p=0.06$; Fig. 2$)$. A ratio of less than 0.5 between the maximum diameter of parenchyma of pancreatic tail and the pancreatic corpus was observed in 3 patients $(17 \%)$ with malignant and 1 patient $(3.6 \%)$ with a benign IPMN ( $p=$ 0.29; Fig. 2). 
Table 2. Components of the scoring system for the evaluation of IMPN in abdominal CT scans

\begin{tabular}{lll}
\hline Parameter & Value & Score \\
\hline Maximum diameter of main pancreatic duct, mm & $<5$ & 0 \\
& $5-10$ & 1 \\
\hline Maximum diameter of cystic formations, mm & $>10$ & 0 \\
\hline Bile duct-obstruction (no/yes) & $>30$ & 1 \\
\hline Presence of solid tumor components or mural nodules (no/yes) & No & 0 \\
\hline Contrast enhancement in cystic walls (no/yes) & Yes & 2 \\
\hline Peripancreatic lymph nodes $>10$ mm (no/yes) & No & 3 \\
\hline Abrupt diameter changes of the main pancreatic duct $>50 \%$ (no/yes) & No & 0 \\
\hline CT-density in the cystic part of the lesion on non-enhanced CT, HU & Yes & 1 \\
\hline
\end{tabular}

\section{Multivariate Regression Analysis}

In a multivariate regression analysis the following were taken as independent variables: the maximum diameter of the main pancreatic duct, the maximum diameter of cystic formations, the presence of bile duct obstruction, the presence of solid tumor components, contrast enhancement in cystic walls, the presence of peripancreatic lymph nodes $>10 \mathrm{~mm}$, abrupt changes of the main pancreatic duct of more than $50 \%$, and the CT density in the cystic part of the lesion on the non-enhanced CT. Only the diameter of the pancreatic duct ( $p=$ $0.0015)$ and the CT density in the cystic part on the nonenhanced CT $(p=0.022)$ showed a significant association, and the association with enlarged peripancreatic lymph nodes almost reached statistical significance $(p=0.050)$. The overall $r^{2}$ value was 0.60 , indicating that $60 \%$ of the probability for malignancy could be explained by the model.

\section{Diagnostic Accuracy of a Summation Score for the \\ CT-Based Prediction of Malignancy in \\ Pancreatic IPMNs}

To create a summation score of predictive factors for the diagnosis of a malignant IMPN, the CT-based parameters exhibiting significant differences between patients with benign and malignant IMPN were selected
(Table 2). In addition, tumor size was included in the score based on the experience from previous studies. Thus, scores from 0 to 3 were assigned to the various components. The relative weight of the individual parameters was based on published recommendations and guidelines and the individual judgment of the investigators.

Details of the scoring system are summarized in Table 2. The final possible scores ranged between 0 and 15. This score yielded the best diagnostic threshold between 2 and 3 points. Thus, the probability of a malignant IPMN in patients with a score of less than 3 points was $10 \%$, whereas the respective probability in patients with a sore of 3 points or more was $94 \%$. On that basis, a final score of 3 or more points was considered to predict malignancy. A diagnostic sensitivity of 0.84 , a specificity of 0.96 , a PPV of 0.94 and an NPV of 0.9 were calculated for this score (Table 3 ).

A summation score of 6 or more points predicted malignancy at an even higher specificity (1.0), albeit with lower sensitivity (0.42; Table 3 ).

The respective diagnostic values of the intermediate summation scores (between 3 and 6 ) for the prediction of malignancy can be derived from Table 3. Overall, the diagnostic accuracy of abdominal CT scans for the detection of malignant IPMN was greatly enhanced by the use 
Table 3. Probability of malignancy for different summation scores determined based on abdominal CT scans

\begin{tabular}{|c|c|c|c|c|c|c|c|}
\hline Number of points & $\begin{array}{l}\text { Number of malignant/ } \\
\text { benign tumors }\end{array}$ & $\begin{array}{l}\text { Probability of } \\
\text { malignancy, \% }\end{array}$ & $\begin{array}{l}\text { Probability of } \\
\text { benignancy, \% }\end{array}$ & Sensitivity & Specificity & PPV & NPV \\
\hline 0 & $0 / 12$ & 0 & 100 & 0 & 0.57 & 0 & 0.46 \\
\hline Less than 3 & $3 / 27$ & 10 & 90 & 0.16 & 0.04 & 0.1 & 0.06 \\
\hline 3 or more & $16 / 1$ & 94 & 6 & 0.84 & 0.96 & 0.94 & 0.9 \\
\hline 6 or more & $8 / 0$ & 100 & 0 & 0.42 & 1 & 1 & 0.72 \\
\hline
\end{tabular}

Diagnostic sensitivity, specificity, positive predictive value (PPV), and negative predictive value (NPV) of different summation scores for the detection of a malignant IPMN are presented.

of the summation score compared to the respective accuracies of the individuals CT-based parameters.

In addition to this score, in which the relative weight of the individual parameters was determined in a subjective fashion by the investigators, 2 other scores were calculated: a "non-weighted" score, in which the same number of points was assigned to each parameter as well as an "objectively weighted score," in which higher scores were assigned only to those parameters that were significantly associated with malignancy in the multivariate regression analysis (details not shown). A comparison of these 3 scoring systems yielded the highest sensitivity and specificity for the subjectively weighted score.

\section{Discussion}

This study was designed to determine the diagnostic accuracy of various CT-based parameters for the prediction of malignancy of pancreatic IPMN. Based on the analysis of 47 scans, the diameter of the main pancreatic duct, bile duct obstruction, solid tissue components, contrast enhancement in cystic walls, enlarged peripancreatic lymph nodes, abrupt diameter changes of the main pancreatic duct, and a high CT density of the lesions were found to be more prevalent in malignant than in benign IPMN. Among those parameters, the diameter of the main pancreatic duct exhibited the highest individual diagnostic accuracy. This parameter, as well as the CT density in the cystic part, also remained significantly associated with malignancy in a multivariate regression analysis. The diagnostic sensitivity and specificity of a weighted summation score comprising 8 different parameters were much higher than the diagnostic sensitivity and specificity of each individual parameter.
Previous studies have suggested a number of individual criteria to predict malignancy in pancreatic IPMN [6, 14-16]. The summation score suggested here was based on the evaluation of these parameters as well as the determination of the CT density of the lesions. Only parameters that were found to be different between patients with benign and malignant IPMN were selected for the summation score. Notably, using this simple scoring system, IPMN carcinomas were predicted with a sensitivity of $84 \%$ and specificity of $96 \%$ at a score of 3 or more. This diagnostic specificity was even greater in lesions with higher scores (1.0 at a score of 6 or more), albeit with less sensitivity. In the present analysis, the maximum diameter of the cystic lesions was not different between benign and malignant IPMN. This finding is at variance with some, but not all previous studies. Indeed, more recent guidelines suggest to observe branch duct IPMN even $>3 \mathrm{~cm}$ if there are no other signs of malignancy [6].

A newly established criterion in this study was the mean CT density of the cystic lesions. This parameter was significantly different between benign and malignant IPMN, although there was still a large degree of overlap between the groups. However, because the determination of CT density involves manual contouring of a region of interest within the lesion, the accurate assessment might depend on the experience of the examiner. Nevertheless, in combination with the other parameters, determining the CT density of the cystic lesions might be a valuable addition to the overall diagnostic evaluation of pancreatic IPMN.

Although it is commonly accepted that the involvement of the main pancreatic duct is strongly associated with malignancy in IPMN, the accurate determination of main duct involvement using CT scan images is quite challenging. Therefore, the dilation of the main pancre- 
atic duct has been used as indirect evidence of main pancreatic ductal involvement in this as well as in previous studies [17-20]. Indeed, this indirect parameter exhibited the strongest individual predictive value for malignancy in pancreatic IPMN.

In this study, only CT scan images were analyzed, even though the diagnostic value of pancreatic MRI or EUS is often superior [21-23]. This approach was chosen because of the broad availability of the CT. Furthermore, the evaluation of pancreatic lesions by EUS is more invasive and largely dependent on the experience of the individual examiner as well as the anatomical conditions of the patient [24]. Limitations of pancreatic MRI include the long duration of the examination as well as movement artefacts [25]. Therefore, abdominal CT scans will likely remain important components of the diagnostic workup for pancreatic IPMN.

Although pancreatic CT scans may therefore add some information for the initial evaluation of pancreatic cystic lesions, such examinations cannot be recommended for the long-term follow-up because of the high radiation exposure. Therefore, MRI imaging is considered the gold standard for the long-term surveillance of pancreatic IPMN as recently emphasized in the American Gastroenterological Association Institute Guidelines $[26,27]$. Alternatively, branch-duct IPMN without highrisk stigmata or worrisome features may safely be followed up by EUS [28]. If those 2 diagnostic options are not available, patients should be referred to a specialized center.

In addition to the differences in various CT-based parameters between patients with benign and malignant IPMN, this study has also revealed higher CA 19-9 levels in the malignant cases, in agreement with previous studies [17, 29-31]. However, the wide overlap in CA 19-9 levels between the groups does not allow for a clear discrimination based on this parameters. Nevertheless, in combination with radiographic and endoscopic findings as well as the patients' symptoms, determination of CA 19-9 might facilitate the diagnosis of malignant IPMN.

In contrast to these CT-based and laboratory parameters, the prevalence patterns of known diabetes and of exocrine pancreatic insufficiency were not different between the groups. This finding is of interest because both factors are known to be associated with the incidence of pancreatic adenocarcinomas [32-34]. In fact, the occurrence of diabetes in lean individuals of advanced age has even been suggested as a diagnostic signal for the presence of pancreatic carcinomas [35]. Mechanistically, the hyperglycemia in patients with pancreatic cancer has been associated with the local destruction of islet cell tissue as well as the release of inflammatory cytokines that act to inhibit beta-cell function [36]. The lack of association between diabetes and malignant IPMN underlines the different pathogenetic mechanisms of pancreatic adenocarcinoma and IPMN. However, the prevalence rates of both diabetes (42.1 vs. $44.4 \%$ ) and exocrine pancreatic insufficiency (82.4 and $100 \%)$ were generally quite high in this group of patients, thereby indicating a large extent of both exocrine and endocrine abnormalities in these patients, irrespective of the dignity of the tumors.

As it is for most retrospective studies, certain limitations apply to the present analysis. Most importantly, in terms of design only IMPN that had been subjected to surgery could be included in this study. This was done out of necessity because the histopathological diagnosis was used as the reference standard. Nevertheless, it cannot be excluded that the results of this study would be different in a selection of tumor cases with less obvious pathological findings that are not routinely subjected to surgery. Therefore, it seems advisable to apply the criteria defined here to a prospective series of pancreatic IPMN to further validate this summation score.

From a clinical perspective, a scoring system such as the one proposed here may aid physicians in determining malignancy in pancreatic IPMN. Such formal classification is certainly not meant to substitute for the individual experience of the radiologists evaluating abdominal CT scans. Clearly, in many cases, the accurate diagnosis can already be derived from the image analysis without such classification. However, for less experienced examiners or in cases of less obvious alterations, a scoring system may help physicians to come to an accurate prediction of malignancy. Considering the importance of this clinical judgment in determining whether patients should be subjected to pancreatic surgery or not, such formal criteria may be a helpful clinical tool. Nevertheless, the overall decision-making process in such cases must consider all information available, including patients' symptoms, laboratory abnormalities, and a combination of different imaging techniques, such as CT, MRI, and EUS.

In conclusion, the present study has revealed a number of CT-based criteria that can help to distinguish between benign and malignant pancreatic IPMN. However, the individual sensitivity and specificity of each of these parameters to detect malignancy was rather low. A greater diagnostic accuracy can be obtained from the determination of a simple summation score consisting of 8 different 
components. Such summation score may aid physicians in determining malignancy in pancreatic IPMN based on abdominal CT scans.

\section{Acknowledgments}

We are indebted to Professor Michael Nauck for help with the statistical analyses.

\section{Disclosure Statement}

The authors report no conflicts of interest in relation to this work.

\section{Funding}

None.

\section{References}

1 Cooper CL, O'Toole SA, Kench JG: Classification, morphology and molecular pathology of premalignant lesions of the pancreas. Pathology 2013;45:286-304.

2 Belyaev O, Seelig MH, Muller CA, Tannapfel A, Schmidt WE, Uhl W: Intraductal papillary mucinous neoplasms of the pancreas. J Clin Gastroenterol 2008;42:284-294

3 Spinelli KS, Fromwiller TE, Daniel RA, Kiely JM, Nakeeb A, Komorowski RA, et al: Cystic pancreatic neoplasms: observe or operate. Ann Surg 2004;239:651-657; discussion 657-659.

4 de Jong K, Nio CY, Hermans JJ, Dijkgraaf MG, Gouma DJ, van Eijck $\mathrm{CH}$, et al: High prevalence of pancreatic cysts detected by screening magnetic resonance imaging examinations. Clin Gastroenterol Hepatol 2010;8: 806-811.

5 Sohn TA, Yeo CJ, Cameron JL, Hruban RH, Fukushima N, Campbell KA, et al: Intraductal papillary mucinous neoplasms of the pancreas: an updated experience. Ann Surg 2004; 239:788-797; discussion 797-799.

6 Tanaka M, Fernandez-del Castillo C, Adsay V, Chari S, Falconi M, Jang JY, et al: International consensus guidelines 2012 for the management of IPMN and MCN of the pancreas. Pancreatology 2012;12:183-197.

7 Uhl W, Belyaev O, Herzog T, Mueller CA, Seelig M, Schmidt W, et al: [Intraductal papillary mucinous neoplasms of the pancreas: pro surgical therapy - pro surveillance]. Z Gastroenterol 2008;46:1290-1297.

8 Khalid A, Brugge W: ACG practice guidelines for the diagnosis and management of neoplastic pancreatic cysts. Am J Gastroenterol 2007;102:2339-2349.

9 Mayerle J, Kraft M, Menges P, Simon P, Ringel J, Partecke LI, et al: [Intraductal papillary mucinous neoplasia: which findings support observation?]. Chirurg 2012;83:123-129.

10 Sugiyama M, Izumisato Y, Abe N, Masaki T, Mori T, Atomi Y: Predictive factors for malignancy in intraductal papillary-mucinous tumours of the pancreas. Br J Surg 2003;90: 1244-1249.

11 Fujino Y, Matsumoto I, Ueda T, Toyama H, Kuroda Y: Proposed new score predicting malignancy of intraductal papillary mucinous neoplasms of the pancreas. Am J Surg 2007; 194:304-307.
12 Shin SH, Han DJ, Park KT, Kim YH, Park JB, Kim SC: Validating a simple scoring system to predict malignancy and invasiveness of intraductal papillary mucinous neoplasms of the pancreas. World J Surg 2010;34:776783.

13 Klöppel G, Solcia E, Longnecker DS, Capella C, Sobin LH: Histological typing of tumours of the exocrine pancreas; in WHO International Histological Classification of Tumours, ed 2. New York, Springer Verlag, Berlin Heidelberg, 1996, pp 12-19.

14 Brambs HJ, Juchems M: Radiologische Diagnostik der intraduktalen papillär muzinösen Neoplasie. Chirurg 2012;83:116-122.

15 Ogawa H, Itoh S, Ikeda M, Suzuki K, Naganawa S: Intraductal papillary mucinous neoplasm of the pancreas: assessment of the likelihood of invasiveness with multisection CT. Radiology 2008;248:876-886.

16 Kato Y, Takahashi S, Gotohda N, Konishi M: Risk factors for malignancy in branched-type intraductal papillary mucinous neoplasms of the pancreas during the follow-up period. World J Surg 2015;39:244-250.

17 Mimura T, Masuda A, Matsumoto I, Shiomi $\mathrm{H}$, Yoshida S, Sugimoto M, et al: Predictors of malignant intraductal papillary mucinous neoplasm of the pancreas. J Clin Gastroenterol 2010;44:e224-e229.

18 Hirano S, Kondo S, Tanaka E, Shichinohe T, Suzuki O, Shimizu M, et al: Role of CT in detecting malignancy during follow-up of patients with branch-type IPMN of the pancreas. Hepatogastroenterology 2009;56:515-518.

19 Gupta R, Mortele KJ, Tatli S, Girshman J, Glickman JN, Levy AD, et al: Pancreatic intraductal papillary mucinous neoplasms: role of CT in predicting pathologic subtypes. AJR Am J Roentgenol 2008;191:1458-1464.

20 Ogawa H, Itoh S, Ikeda M, Suzuki K, Naganawa S: Intraductal papillary mucinous neoplasm of the pancreas: assessment of the likelihood of invasiveness with multisection CT. Radiology 2008;248:876-886.

21 Schrader H, Wiese M, Ellrichmann M, Belyaev O, Uhl W, Tannapfel A, et al: Diagnostic value of quantitative EUS elastography for malignant pancreatic tumors: relationship with pancreatic fibrosis. Ultraschall Med 2012;33:E196-E201.
22 Rafique A, Freeman S, Carroll N: A clinical algorithm for the assessment of pancreatic lesions: utilization of 16- and 64-section multidetector CT and endoscopic ultrasound. Clin Radiol 2007;62:1142-1153.

23 Tirkes T, Menias CO, Sandrasegaran K: MR imaging techniques for pancreas. Radiol Clin North Am 2012;50:379-393.

24 Gardner TB, Gordon SR: Interobserver agreement for pancreatic endoscopic ultrasonography determined by same day back-to-back examinations. J Clin Gastroenterol 2011;45: 542-545.

25 Choi JY, Lee JM, Lee MW, Kim SJ, Choi SY, Kim JY, et al: Magnetic resonance pancreatography: comparison of two- and three-dimensional sequences for assessment of intraductal papillary mucinous neoplasm of the pancreas. Eur Radiol 2009;19:2163-2170.

26 Vege SS, Ziring B, Jain R, Moayyedi P; Clinical Guidelines Committee; American Gastroenterology Association: American gastroenterological association institute guideline on the diagnosis and management of asymptomatic neoplastic pancreatic cysts. Gastroenterology 2015;148:819-822; quiz e12-e13.

27 Scheiman JM, Hwang JH, Moayyedi P: American gastroenterological association technical review on the diagnosis and management of asymptomatic neoplastic pancreatic cysts. Gastroenterology 2015;148:824848.e22.

28 Lawson RD, Hunt GC, Giap AQ, Krinsky ML, Slezak J, Tang RS, et al: Pancreatic cysts suspected to be branch duct intraductal papillary mucinous neoplasm without concerning features have low risk for development of pancreatic cancer. Ann Gastroenterol 2015;28:487-494.

29 Hirono S, Tani M, Kawai M, Okada K, Miyazawa M, Shimizu A, et al: The carcinoembryonic antigen level in pancreatic juice and mural nodule size are predictors of malignancy for branch duct type intraductal papillary mucinous neoplasms of the pancreas. Ann Surg 2012;255:517-522.

30 Fritz S, Hackert T, Hinz U, Hartwig W, Buchler MW, Werner J: Role of serum carbohydrate antigen 19-9 and carcinoembryonic antigen in distinguishing between benign and invasive intraductal papillary mucinous neoplasm of the pancreas. Br J Surg 2011;98:104-110. 
31 Ingkakul T, Sadakari Y, Ienaga J, Satoh N, Takahata S, Tanaka M: Predictors of the presence of concomitant invasive ductal carcinoma in intraductal papillary mucinous neoplasm of the pancreas. Ann Surg 2010;251:70-75.

32 Menge BA, Schrader H, Breuer TG, Dabrowski Y, Uhl W, Schmidt WE, et al: Metabolic consequences of a $50 \%$ partial pancreatectomy in humans. Diabetologia 2009;52:306-317.
33 Pannala R, Basu A, Petersen GM, Chari ST: New-onset diabetes: a potential clue to the early diagnosis of pancreatic cancer. Lancet Oncol 2009; 10:88-95.

34 Schrader H, Menge BA, Belyaev O, Uhl W, Schmidt WE, Meier JJ: Amino acid malnutrition in patients with chronic pancreatitis and pancreatic carcinoma. Pancreas 2009;38:416421.
35 Hart PA, Chari ST: Diabetes mellitus and pancreatic cancer: why the association matters? Pancreas 2013;42:1207-1209.

36 Chari ST, Zapiach M, Yadav D, Rizza RA: Beta-cell function and insulin resistance evaluated by HOMA in pancreatic cancer subjects with varying degrees of glucose intolerance. Pancreatology 2005;5:229233

\section{Erratum}

In the article by Floer $\mathrm{M}$ und Meister T, entitled "Endoscopic improvement of the adenoma detection rate during colonoscopy - where do we stand in 2015?" [Digestion 2016;93:202-213, DOI: 10.1159/000442464], the EndoRings seen on Figure 3 was attached from the wrong direction. The corrected figure is shown below:
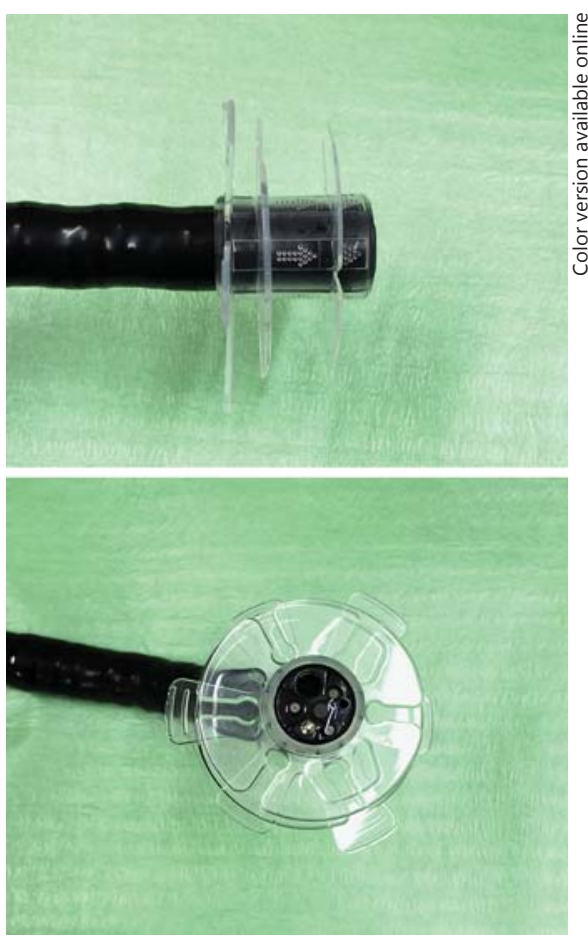

Fig. 3. EndoRings endoscopic add-on device distally attached to the colonoscope (lateral and forward view). 